\title{
Hypercontractivity of simple random variables
}

\author{
by \\ PAWee WolfF (Warszawa)
}

\begin{abstract}
The optimal hypercontractivity constant for a natural operator semigroup acting on a discrete finite probability space is established up to a universal factor. The two-point spaces are proved to be the extremal case. The constants obtained are also optimal in the related moment inequalities for sums of independent random variables.
\end{abstract}

1. Notation and preliminary results. Let $\Omega$ be an arbitrary finite set and let $\mu$ be a probability measure on the measurable space $\left(\Omega, 2^{\Omega}\right)$. For any function $f: \Omega \rightarrow \mathbb{R}, E_{\mu} f$ denotes the expected value of $f$ with respect to $\mu$. To avoid trivialities, we assume that $\mu$ has at least two atoms with non-zero measure.

We shall consider operators acting on the finite-dimensional space $L_{2}(\mu)$ (for abbreviation, we write $L_{p}(\mu)$ instead of $L_{p}(\Omega, \mu)$ ) over the real scalar field. Let $L$ be the orthogonal projection in $L_{2}(\mu)$ onto the subspace of functions with zero mean, i.e. $L=\mathrm{Id}-E_{\mu}$. We define the semigroup $T_{t}=$ $e^{-t L}(t \geq 0)$ of self-adjoint operators. Throughout the paper, we shall use the explicit formula

$$
T_{t}=E_{\mu}+e^{-t} L=\left(1-e^{-t}\right) E_{\mu}+e^{-t} \mathrm{Id},
$$

which implies that $\left(T_{t}\right)_{t \geq 0}$ is a non-negativity preserving semigroup of contractions from $L_{p}(\mu)$ to $L_{p}(\mu)$ for $1 \leq p \leq \infty$.

Definition 1.1. The semigroup $\left(T_{t}\right)_{t \geq 0}$ is $(p, q)$-hypercontractive (for $1<q<p<\infty)$ if there exists $t_{0} \geq 0$ such that for all $t \geq t_{0}$ and $f \in L_{q}(\mu)$,

$$
\left\|T_{t} f\right\|_{L_{p}(\mu)} \leq\|f\|_{L_{q}(\mu)} .
$$

If such a $t_{0}$ exists and is the least possible (the minimum exists, since obviously $T_{t}$ is a strongly continuous semigroup), then $\sigma_{p, q}(\mu):=e^{-t_{0}}$ is the

2000 Mathematics Subject Classification: Primary 60E15.

Key words and phrases: discrete measure, hypercontractivity, moment inequality, simple random vector.

Research partially supported by MEiN Grant 1 PO3A 01229. 
$(p, q)$-hypercontractivity constant for the measure $\mu$. (Non-triviality of $\mu$ gives $t_{0}>0$, i.e., $\sigma_{p, q}(\mu) \in(0,1)$.)

Note that hypercontractivity of the semigroup $\left(T_{t}\right)_{t \geq 0}$ does not depend on the set $\Omega$ itself, but only on the weights of the atoms of the measure $\mu$. In particular, if $\# \Omega=2$, then we may assume that $\mu=\beta \delta_{-\alpha}+\alpha \delta_{\beta}$ for some $\alpha \in(0,1 / 2]$ with $\beta=1-\alpha$. Therefore we shall use the notation

$$
\sigma_{p, q}(\alpha):=\sigma_{p, q}\left(\beta \delta_{-\alpha}+\alpha \delta_{\beta}\right) .
$$

REMARK 1.1. The standard duality argument yields $\sigma_{p, q}(\mu)=\sigma_{q^{\prime}, p^{\prime}}(\mu)$, where $p^{\prime}=p /(p-1)$ and $q^{\prime}=q /(q-1)$. Indeed, self-adjointness of $T_{t}$ implies that $\left\|T_{t}\right\|_{L_{q}(\mu) \rightarrow L_{p}(\mu)}=\left\|T_{t}\right\|_{L_{p^{\prime}}(\mu) \rightarrow L_{q^{\prime}}(\mu)}$.

REMARK 1.2. For $f=a+g$, where $a \in \mathbb{R}$ and $E_{\mu} g=0$, we have $T_{t} f=a+e^{-t} g$. Since $T_{t}$ preserves non-negativity, we have $T_{t}|f| \geq T_{t} f$ and $T_{t}|-f| \geq-T_{t} f$, so $T_{t}|f| \geq\left|T_{t} f\right|$. Therefore in order to check (1.1) we can consider only $f=a+g \geq 0$. Then, except the trivial case $g \equiv 0$, we must have $a>0$ and, by homogeneity, we can assume $a=1$. Therefore

$$
\begin{aligned}
\sigma_{p, q}(\mu)=\max \{\sigma \in(0,1): \| 1 & +\sigma g\left\|_{L_{p}(\mu)} \leq\right\| 1+g \|_{L_{q}(\mu)} \\
& \text { whenever } \left.E_{\mu} g=0 \text { and } 1+g \geq 0\right\}
\end{aligned}
$$

and in the case of the two-point measure $\mu=\beta \delta_{-\alpha}+\alpha \delta_{\beta}$ every $g$ is of the form $g(x)=x u$ for $u \in[-1 / \beta, 1 / \alpha]$.

The hypercontractivity of semigroups has been transferred to the context of random variables. Let us recall the following definition introduced by Krakowiak and Szulga [5] (see also [6] and [7, Ch. 3]).

Definition 1.2. Let $0<q<p<\infty$, let $(\mathbf{F},\|\cdot\|)$ be a separable Banach space and let $X$ be a random vector with values in $\mathbf{F}$ such that $E\|X\|^{p}<\infty$. Then we say that $X$ is $(p, q)$-hypercontractive with constant $\sigma \in(0,1)$ if

$$
\forall v \in \mathbf{F} \quad\|v+\sigma X\|_{p} \leq\|v+X\|_{q} .
$$

(The notation $\|Y\|_{p}$ means $\left(E\|Y\|^{p}\right)^{1 / p}$.)

Remark 1.3. Let $X$ be a zero-mean $\mathbf{F}$-valued random vector which is simple (i.e. takes on finitely many values) and $\mu$ be its distribution. A standard reasoning shows that if the semigroup $T_{t}=e^{-t\left(\operatorname{Id}-E_{\mu}\right)}$ is $(p, q)$ hypercontractive, then $X$ is $(p, q)$-hypercontractive with constant $\sigma_{p, q}(\mu)$. Indeed, taking $e^{-t_{0}}=\sigma:=\sigma_{p, q}(\mu)$ we have $\left\|T_{t_{0}}\right\|_{L_{q}(\mu) \rightarrow L_{p}(\mu)} \leq 1$. Considering, for a fixed $v \in \mathbf{F}$, the convex function $f(x)=\|v+x\|$ and using the Jensen inequality we obtain

$$
\begin{aligned}
& \quad\left(T_{t_{0}} f\right)(X)=(1-\sigma) E f(X)+\sigma f(X) \geq(1-\sigma) f(0)+\sigma f(X) \geq f(\sigma X), \\
& \text { so }\|v+\sigma X\|_{p}=\|f(\sigma X)\|_{p} \leq\left\|\left(T_{t_{0}} f\right)(X)\right\|_{p} \leq\|f(X)\|_{q}=\|v+X\|_{q}
\end{aligned}
$$

Let us recall the following result $[6,7]$ : 
Proposition 1.1. Let $0<q<p<\infty$. If $\left(X_{k}\right)_{k=1}^{n}$ is a sequence of independent random vectors with values in a separable Banach space $\mathbf{F}$ and each $X_{k}$ is $(p, q)$-hypercontractive with the same constant $\sigma$, then

$$
\forall v \in \mathbf{F} \quad\|v+\sigma S\|_{p} \leq\|v+S\|_{q}
$$

where $S=\sum_{k=1}^{n} X_{k}$. In particular,

$$
\|S\|_{p} \leq \sigma^{-1}\|S\|_{q}
$$

We shall use the following notation. For $a, b \in \mathbb{R}, a \vee b=\max (a, b)$, $a \wedge b=\min (a, b)$. If $x, y \geq 0$ and $c \geq 1$ are such that $c^{-1} x \leq y \leq c x$, then we shall write $x \stackrel{c}{\sim} y$. We shall often omit the constant $c$ and write $x \sim y$ whenever $c$ is some universal (numerical) constant. For $x, y \geq 0, x \lesssim y$ means that $x \leq c y$, where $c>0$ is a universal constant.

In Section 2 we formulate known and new results on the constants $\sigma_{p, q}(\alpha)$. Proofs of the latter are presented in Section 4. In Section 3 we show that $\sigma_{p, q}(\mu)$ are comparable with $\sigma_{p, q}\left(\alpha_{*}\right)$, where $\alpha_{*}$ is the mass of the least atom of $\mu$. This solves a problem posed by Janson [4].

2. Hypercontractivity constants for two-point measures. It is a classical result $[2,1]$ that for $1<q<p<\infty$,

$$
\sigma_{p, q}(1 / 2)=\sqrt{\frac{q-1}{p-1}} .
$$

The values of $\sigma_{2, q}(\alpha)$ and $\sigma_{p, 2}(\alpha)$ for $\alpha \in(0,1 / 2)$ and $1<q<2<p<\infty$ were established by Oleszkiewicz [10]:

$$
\sigma_{2, q}(\alpha)=\left(\frac{\beta^{2-2 / q}-\alpha^{2-2 / q}}{\alpha^{1-2 / q} \beta-\beta^{1-2 / q} \alpha}\right)^{1 / 2}, \quad \sigma_{p, 2}(\alpha)=\left(\frac{\beta^{2 / p}-\alpha^{2 / p}}{\alpha^{2 / p-1} \beta-\beta^{2 / p-1} \alpha}\right)^{1 / 2}
$$

where $\beta=1-\alpha$. (One can easily check that $\lim _{\alpha \rightarrow 1 / 2^{-}} \sigma_{2, q}(\alpha)=\sigma_{2, q}(1 / 2)$ and $\lim _{\alpha \rightarrow 1 / 2^{-}} \sigma_{p, 2}(\alpha)=\sigma_{p, 2}(1 / 2)$.) The above results imply that $\sigma_{p, q}(\alpha)$ is well-defined for all $1<q<p<\infty$ (i.e. the related semigroup is $(p, q)$ hypercontractive), since $\sigma_{p, q}(\alpha) \geq \sigma_{2, q}(\alpha)$ for $p \leq 2, \sigma_{p, q}(\alpha) \geq \sigma_{p, 2}(\alpha)$ for $q \geq 2$, and $\sigma_{p, q}(\alpha) \geq \sigma_{2, q}(\alpha) \sigma_{p, 2}(\alpha)$ for $q<2<p$. Elementary calculations show the asymptotic behaviour of $\sigma_{2, q}(\alpha)$ (and $\sigma_{p, 2}(\alpha)$ ) as $\alpha \rightarrow 0$ or $q \rightarrow 1$ $(p \rightarrow \infty)$ (for more details see [10, proof of Theorem 2.1]):

$$
\begin{aligned}
\sigma_{2, q}(\alpha) & \sim \begin{cases}\alpha^{1 / q-1 / 2} & \text { for } \frac{1}{q-1} \leq \ln \frac{1}{\alpha}, \\
\sqrt{(q-1) \alpha \ln (1 / \alpha)} & \text { for } \frac{1}{q-1}>\ln \frac{1}{\alpha},\end{cases} \\
\sigma_{p, 2}(\alpha) & \sim \begin{cases}\alpha^{1 / 2-1 / p} & \text { for } p \leq \ln \frac{1}{\alpha}, \\
\sqrt{(1 / p) \alpha \ln (1 / \alpha)} & \text { for } p>\ln \frac{1}{\alpha} .\end{cases}
\end{aligned}
$$


In Section 3 we will need the following

Lemma 2.1. For $1<q<2<p<\infty$, the functions $(0,1 / 2] \ni \alpha \mapsto$ $\sigma_{2, q}(\alpha)$ and $(0,1 / 2] \ni \alpha \mapsto \sigma_{p, 2}(\alpha)$ are strictly increasing.

We postpone the proof of Lemma 2.1 and of other results stated in this section until Section 4.

The main result of this section deals with estimates of $\sigma_{p, q}(\alpha)$ for all combinations of parameters $\alpha, p, q$ except the case $q<2<p$.

Theorem 2.1. For all $1<q<p \leq 2$ and $\alpha \in(0,1 / 2]$ we have $\sigma_{p, q}(\alpha) \sim$ $\tilde{\sigma}_{p, q}(\alpha)$, where



$$
\begin{aligned}
& \text { for } \alpha \in I_{2}=\left\{\alpha: \frac{e^{1 /(p-1)}}{q-1} \leq \frac{1}{\alpha} \ln \frac{1}{\alpha}<\frac{e^{1 /(q-1)}}{q-1}\right\}, \\
& \tilde{\sigma}_{p, q}(\alpha)=\left\{\frac{q-1}{p-1} \frac{\ln (1 / \alpha)}{1+\ln \left(\frac{q-1}{p-1} \frac{1}{\alpha} \ln \frac{1}{\alpha}\right)}\right. \\
& \text { for } \alpha \in I_{3}=\left\{\alpha: \frac{p-1}{q-1} \leq \frac{1}{\alpha} \ln \frac{1}{\alpha}<\frac{e^{1 /(p-1)}}{q-1}\right\} \text {, } \\
& \sqrt{\frac{q-1}{p-1} \alpha \ln (1 / \alpha)} \quad \text { for } \alpha \in I_{4}=\left\{\alpha: \frac{1}{\alpha} \ln \frac{1}{\alpha}<\frac{p-1}{q-1}\right\} \text {. }
\end{aligned}
$$

As a corollary we obtain

Theorem 2.2. For all $2 \leq q<p<\infty$ and $\alpha \in(0,1 / 2]$ we have $\sigma_{p, q}(\alpha) \sim \widetilde{\sigma}_{p, q}(\alpha)$, where

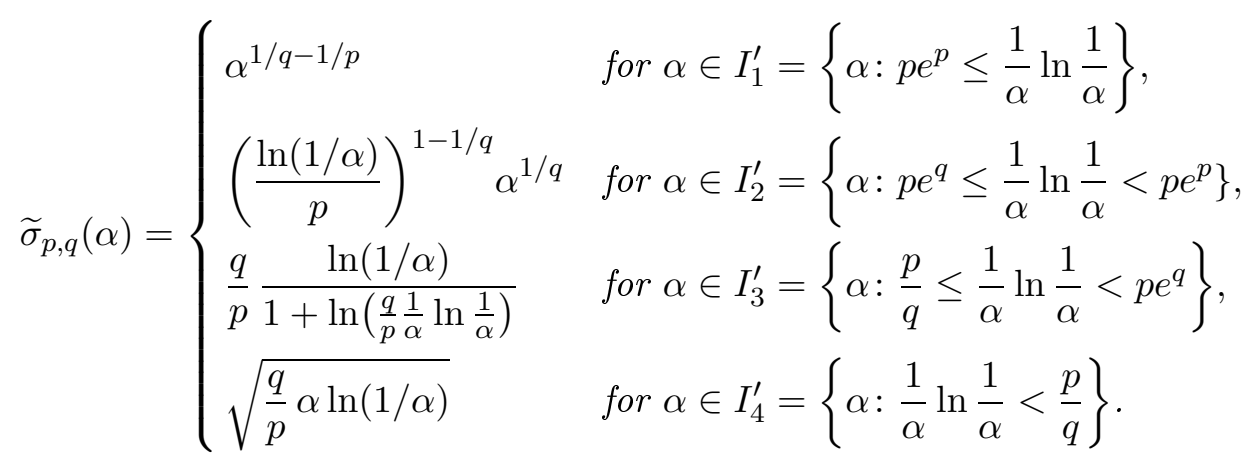

As in the case of the constants $\sigma_{2, q}(\alpha)$ and $\sigma_{p, 2}(\alpha)$, in Section 3 we will use the following

Lemma 2.2. For all $p, q$ such that $1<q<p \leq 2$ or $2 \leq q<p<\infty$ we have $\sigma_{p, q}\left(\alpha_{1}\right) \lesssim \sigma_{p, q}\left(\alpha_{2}\right)$ for all $0<\alpha_{1}<\alpha_{2} \leq 1 / 2$. 
By applying results from [8], we may observe that up to a universal factor, the constants $\widetilde{\sigma}_{p, q}(\alpha)$ appear as a ratio of the $q$ th and $p$ th moments of certain sums of independent two-point random variables.

THEOREM 2.3. Let $\left(X_{k}\right)_{k=1}^{\infty}$ be a sequence of independent random variables with a common distribution $\beta \delta_{-\alpha}+\alpha \delta_{\beta}$, where $\alpha+\beta=1, \alpha \in(0,1 / 2]$. Set $n(\alpha, p)=\lceil p / \ln (1 / \alpha)\rceil$. Then for all $2 \leq q<p<\infty$ and for $S=$ $\sum_{k=1}^{n(\alpha, p)} X_{k}$ we have

$$
\|S\|_{q} \sim \widetilde{\sigma}_{p, q}(\alpha)\|S\|_{p}
$$

On the other hand, by Theorem 2.2, Remark 1.3 and Proposition 1.1, for any sum $S=\sum_{k=1}^{n} v_{k} X_{k}$ where $\left(v_{k}\right)_{k=1}^{n}$ is a sequence in any Banach space $\mathbf{F}$, we have

$$
\|S\|_{q} \gtrsim \widetilde{\sigma}_{p, q}(\alpha)\|S\|_{p}
$$

Thus we see that the case $n=\lceil p / \ln (1 / \alpha)\rceil$ and $v_{1}=\cdots=v_{n} \neq 0$ (or even just $\mathbf{F}=\mathbb{R}$ and $v_{1}=\cdots=v_{n}=1$ ) is extremal up to some universal constant. It is interesting that $n$ depends only on $\alpha$ and $p$ and does not depend on $q$. Moreover, $n$ can be defined as the greatest integer for which $\left\|\sum_{k=1}^{n} X_{k}\right\|_{p} \stackrel{c}{\sim}\left\|\sum_{k=1}^{n} X_{k}\right\|_{\infty}$ with some absolute constant $c>0$ (see the proof of Theorem 2.3).

\section{Hypercontractivity constants for discrete measures}

THEOREM 3.1. Let $\mu$ be a discrete measure with the mass of the least atom equal to $\alpha_{*}>0$. Then for all $1<q<p<\infty$ we have

$$
\inf _{\alpha \in\left[\alpha_{*}, 1 / 2\right]} \sigma_{p, q}(\alpha) \leq \sigma_{p, q}(\mu) \leq \sigma_{p, q}\left(\alpha_{*}\right) .
$$

Proof. The second inequality is quite obvious (e.g. see (1.2)). From now on, we consider only the case $p \geq 2$; the case $1<q<p<2$ follows from the previous one and a duality argument (see Remark 1.1). In order to prove the first inequality we shall prove that for all $t \geq 0$ we have

$$
\left\|T_{t}\right\|_{L_{q}(\mu) \rightarrow L_{p}(\mu)} \leq \sup _{\alpha \in\left[\alpha_{*}, 1 / 2\right]}\left\|S_{t}^{(\alpha)}\right\|_{L_{q}\left(\nu^{(\alpha)}\right) \rightarrow L_{p}\left(\nu^{(\alpha)}\right)}
$$

where $\left(T_{t}\right)_{t \geq 0}$ is the semigroup attached to $\mu$ and $\left(S_{t}^{(\alpha)}\right)_{t \geq 0}$ is the semigroup associated to the two-point measure $\nu^{(\alpha)}=\beta \delta_{-\alpha}+\alpha \delta_{\beta}(\alpha+\beta=1)$. The argument used below is similar to one presented by Diaconis and Saloff-Coste [3] and originates in Rothaus [11]. The main difference is the context-we deal with hypercontractivity, whereas in the cited papers this argument was used to prove logarithmic Sobolev inequalities.

For fixed $t \geq 0$, set

$$
A=\left\|T_{t}\right\|_{L_{q}(\mu) \rightarrow L_{p}(\mu)}=\sup \left\{\left\|T_{t} f\right\|_{L_{p}(\mu)}: f \in L_{q}(\mu),\|f\|_{L_{q}(\mu)}=1\right\} .
$$


Clearly, if $A=1$ then (3.1) is trivial. So assume $A>1$. The fact that $L_{q}(\mu)$ has finite dimension implies that $A<\infty$ and there exists $f_{0}$ for which the above supremum is attained. Defining the functionals $I(f)=\left\|T_{t} f\right\|_{L_{p}(\mu)}^{p}$ and $J(f)=\|f\|_{L_{q}(\mu)}^{q}$ on $L_{q}(\mu)$, we see that $f_{0}$ maximizes $I$ subject to the constraint $J(f)=1$. The functionals $I$ and $J$ are both $C^{1}$ and $D J\left(f_{0}\right) \neq 0$, since $J\left(f_{0}\right)=1$. Therefore for some constant $\lambda$ we have

$$
D I\left(f_{0}\right)=\lambda D J\left(f_{0}\right) .
$$

Since $T_{t}$ preserves non-negativity, we may choose $f_{0}$ to be non-negative. The assumption $A>1$ implies that $f_{0}$ cannot be a constant function. We shall show that $f_{0}$ takes at most two values. The computations of the derivatives from (3.2) are as follows $\left(\langle f, g\rangle_{\mu}\right.$ stands for $\left.E_{\mu} f g\right)$ :

$$
\begin{aligned}
D J\left(f_{0}\right) & =q\left\langle f_{0}^{q-1}, \cdot\right\rangle_{\mu}, \\
D I\left(f_{0}\right) & =D\|\cdot\|_{L_{p}(\mu)}^{p}\left(T_{t} f_{0}\right) \circ D T_{t}\left(f_{0}\right)=p\left\langle\left(T_{t} f_{0}\right)^{p-1}, \cdot\right\rangle_{\mu} \circ T_{t} \\
& =p\left\langle\left(T_{t} f_{0}\right)^{p-1}, T_{t}(\cdot)\right\rangle_{\mu}=p\left\langle T_{t}\left(\left(T_{t} f_{0}\right)^{p-1}\right), \cdot\right\rangle_{\mu}
\end{aligned}
$$

(the last equality follows from the fact that $T_{t}$ is a self-adjoint operator). Thus by (3.2) we have

$$
T_{t}\left(\left(T_{t} f_{0}\right)^{p-1}\right)=\tilde{\lambda} f_{0}^{q-1}
$$

for some constant $\tilde{\lambda}$. Since $f_{0} \geq 0$, also $g_{0}:=T_{t} f_{0} \geq 0$. We have $f_{0}=$ $\left(T_{t}\right)^{-1} g_{0}=e^{t} g_{0}+\left(1-e^{t}\right) E_{\mu} g_{0}$, so

$$
\left(e^{-t} g_{0}^{p-1}+\left(1-e^{-t}\right)\left\|g_{0}\right\|_{L_{p-1}(\mu)}^{p-1}\right)^{1 /(q-1)}=\widetilde{\lambda}\left(e^{t} g_{0}+\left(1-e^{t}\right) E_{\mu} g_{0}\right) .
$$

Fixing an argument $x$ of $g_{0}$ and putting $u:=g_{0}(x) \in[0, \infty)$, we obtain

$$
\left(\left(a u^{p-1}+b v^{p-1}\right)^{1 /(p-1)}\right)^{(p-1) /(q-1)}=c u-d
$$

for some constants $a>0, b, v \geq 0$ and $c, d \in \mathbb{R}$ which do not depend on the choice of $x$. The right hand side of (3.3) is a linear function of $u$, whereas the left hand side of (3.3) is a strictly convex function of $u$. Indeed, it is the composition of the convex and strictly increasing function $[0, \infty) \ni u \mapsto$ $\left(a u^{p-1}+b v^{p-1}\right)^{1 /(p-1)}$ (this expression is just some $L_{p-1}$-norm because we have assumed $p \geq 2$ ) and the function $[0, \infty) \ni z \mapsto z^{(p-1) /(q-1)}$, which is strictly convex and strictly increasing. Therefore the equation (3.3) has at most two solutions $u \in[0, \infty)$, so $g_{0}$ (and also $f_{0}$ ) takes on at most two values.

Therefore $f_{0}$ takes on exactly two different values. Denote them by $u_{1}$, $u_{2}$ so that $\mu\left(f_{0}^{-1}\left(u_{1}\right)\right)=\alpha$ and $\mu\left(f_{0}^{-1}\left(u_{2}\right)\right)=1-\alpha$ for some $\alpha \in(0,1 / 2]$. Clearly, $\alpha \in\left[\alpha_{*}, 1 / 2\right]$ and $\left\|T_{t}\right\|_{L_{q}(\mu) \rightarrow L_{p}(\mu)}=\left\|S_{t}^{(\alpha)}\right\|_{L_{q}\left(\nu^{(\alpha)}\right) \rightarrow L_{p}\left(\nu^{(\alpha)}\right)}$, which proves (3.1). 
Corollary 3.1. Let $\mu$ be a discrete measure with the mass of the least atom equal to $\alpha \in(0,1 / 2)$. Then for all $1<q<2<p<\infty$ we have

$$
\sigma_{2, q}(\mu)=\sigma_{2, q}(\alpha) \quad \text { and } \quad \sigma_{p, 2}(\mu)=\sigma_{p, 2}(\alpha),
$$

and for all $1<q<p<\infty$ satisfying $q, p \leq 2$ or $q, p \geq 2$ we have

$$
\sigma_{p, q}(\mu) \sim \sigma_{p, q}(\alpha) .
$$

Proof. The first part follows from Theorem 3.1 and Lemma 2.1, whereas the second part follows from Theorem 3.1 and Lemma 2.2.

It is worth mentioning that Theorem 3.1 allows us to generalize some Sobolev-type inequality considered by Latała and Oleszkiewicz [9, Remark 2] from a two-point space to any finite space.

Corollary 3.2. Let $\mu$ be a discrete measure with the mass of the least atom equal to $\alpha \in(0,1 / 2), \beta=1-\alpha, 1<q<2$. Define the quadratic form $\mathcal{E}(f)=E_{\mu} f L f$, where $L=\mathrm{Id}-E_{\mu}$. Then for every function $f \in L_{2}(\mu)$,

$$
E_{\mu} f^{2}-\left(E_{\mu}|f|^{q}\right)^{2 / q} \leq C_{q}(\alpha) \mathcal{E}(f)
$$

where

$$
C_{q}(\alpha)=\frac{\alpha^{1-2 / q}-\beta^{1-2 / q}}{\alpha^{1-2 / q} \beta-\beta^{1-2 / q} \alpha} .
$$

Proof. Consider the self-adjoint semigroup $T_{t}=e^{-t L}$. Corollary $3.1 \mathrm{im}-$ plies that if $t_{0}$ satisfies $e^{-t_{0}}=\sigma_{2, q}(\alpha)$ then $\left\|T_{t_{0}}\right\|_{L_{q}(\mu) \rightarrow L_{2}(\mu)} \leq 1$, hence

$$
\begin{aligned}
\|f\|_{L^{q}(\mu)}^{2} & \geq\left\|T_{t_{0}} f\right\|_{L^{2}(\mu)}^{2}=\left\langle T_{t_{0}} f, T_{t_{0}} f\right\rangle_{\mu}=\left\langle f, T_{2 t_{0}} f\right\rangle_{\mu} \\
& =e^{-2 t_{0}} E_{\mu} f^{2}+\left(1-e^{-2 t_{0}}\right)\left(E_{\mu} f\right)^{2}=E_{\mu} f^{2}-\left(1-e^{-2 t_{0}}\right) \mathcal{E}(f) \\
& =E_{\mu} f^{2}-C_{q}(\alpha) \mathcal{E}(f),
\end{aligned}
$$

because, as one may check, $C_{q}(\alpha)=1-\sigma_{2, q}^{2}(\alpha)$.

Letting $q \rightarrow 2$ in the inequality (3.4) one can obtain the logarithmic Sobolev inequality considered in [3, Theorem A.1]:

$$
E_{\mu} f^{2} \ln f^{2}-E_{\mu} f^{2} \ln E_{\mu} f^{2} \leq \frac{\ln \beta-\ln \alpha}{\beta-\alpha} \mathcal{E}(f) .
$$

By Corollary 3.1, Remark 1.3 and Proposition 1.1 we finally get

CoROllaRY 3.3. Let $\left(X_{k}\right)_{k=1}^{n}$ be a sequence of independent simple random vectors with values in a Banach space $\mathbf{F}$, and $S=\sum_{k=1}^{n} X_{k}$. Let $\alpha=\min \left\{P\left(X_{k}=v\right) \neq 0: v \in \mathbf{F}\right\}$. Then for every $2 \leq q<p<\infty$,

$$
\|S\|_{p} \leq C \sigma_{p, q}^{-1}(\alpha)\|S\|_{q}
$$

where $C>0$ is a universal constant, which may be 1 if $q=2$. 
Moreover, by Theorem 2.2 we clearly get $\|S\|_{p} \lesssim \widetilde{\sigma}_{p, q}^{-1}(\alpha)\|S\|_{q}$, whereas Theorem 2.3 implies that for fixed $\alpha, p, q$ the constants in the above moment inequalities are optimal up to some universal factor.

\section{Proofs}

Proof of Lemma 2.1. Due to Remark 1.1 it suffices to prove that $f(\alpha)=$ $\sigma_{2, q}^{2}(\alpha)$ is increasing for $\alpha \in(0,1 / 2)$. For $x, y>0, x \neq y$ and $t \in(0,1)$, we define

$$
\varphi(x, y)=\frac{x^{1-t}-y^{1-t}}{x y^{-t}-y x^{-t}} .
$$

Clearly, $\varphi(x, y)=\varphi(y, x)$ and $\varphi(x, y)=\varphi(\lambda x, \lambda y)$ for $\lambda>0$. Putting $t=$ $2 / q-1$ we have $f(\alpha)=\varphi(1-\alpha, \alpha)$, thus

$$
\begin{aligned}
f^{\prime}(\alpha) & =\partial_{y} \varphi(1-\alpha, \alpha)-\partial_{x} \varphi(1-\alpha, \alpha) \\
& =\frac{1}{\alpha} \partial_{x} \varphi\left(\frac{1-\alpha}{\alpha}, 1\right)-\frac{1}{1-\alpha} \partial_{x} \varphi\left(\frac{\alpha}{1-\alpha}, 1\right) .
\end{aligned}
$$

Therefore the proof will be completed by showing that

$$
\partial_{x} \varphi(x, 1)>0 \quad \text { for } x \in(0,1) \quad \text { and } \quad \partial_{x} \varphi(x, 1)<0 \quad \text { for } x \in(1, \infty) .
$$

Putting

$$
\psi(x, s)=\frac{1}{s}\left(x^{s}-x^{-s}\right)=\frac{\ln x}{s} \int_{0}^{s}\left(x^{u}+x^{-u}\right) d u
$$

for $x>0$ and $s \in(0,1]$, we have

$$
\begin{aligned}
\partial_{x} \varphi(x, 1) & =\frac{1}{\left(x-x^{-t}\right)^{2}}\left((1-t) x^{-t}\left(x-x^{-t}\right)-\left(x^{-t+1}-1\right)\left(1+t x^{-t-1}\right)\right) \\
& =\frac{t x^{-t}}{\left(x-x^{-t}\right)^{2}}(\psi(x, t)-\psi(x, 1)) .
\end{aligned}
$$

Now it suffices to show that $\psi(x, s)$ is increasing as a function of $s \in(0,1]$ for $x>1$ and decreasing for $x \in(0,1)$. The former statement implies the latter, because $\psi(x, s)=-\psi(1 / x, s)$. Therefore we consider the case $x>1$. Fix $0<s_{1}<s_{2} \leq 1$ and put $\lambda=s_{1} / s_{2}<1$. Using the inequality $x^{\lambda u}+x^{-\lambda u}<$ $x^{u}+x^{-u}$, which holds by the convexity of $u \mapsto x^{u}$, we obtain

$$
\begin{aligned}
\psi\left(x, s_{1}\right) & =\frac{\ln x}{s_{1}} \int_{0}^{s_{2}}\left(x^{\lambda u}+x^{-\lambda u}\right) \lambda d u \\
& <\frac{\ln x}{s_{2}} \int_{0}^{s_{2}}\left(x^{u}+x^{-u}\right) d u=\psi\left(x, s_{2}\right) .
\end{aligned}
$$


Now, we turn to the proof of Theorem 2.1. First, note that Remark 1.2 implies that $\sigma_{p, q}(\alpha)$ is the greatest $\sigma$ such that

$$
\left(\beta|1-\sigma \alpha u|^{p}+\alpha|1+\sigma \beta u|^{p}\right)^{1 / p} \leq\left(\beta|1-\alpha u|^{q}+\alpha|1+\beta u|^{q}\right)^{1 / q} \quad(\beta=1-\alpha)
$$

for all $u \in[-1 / \beta, 1 / \alpha]$ (or, equivalently, for all $u \in \mathbb{R}$ ).

The following lemmas will be used in the proof of Theorem 2.1.

Lemma 4.1. For all $1<q<p<\infty$ and $0<\alpha_{1}<\alpha_{2} \leq 1 / 2$ with $\beta_{i}=1-\alpha_{i}(i=1,2)$ we have $\sigma_{p, q}\left(\alpha_{1}\right) \stackrel{c}{\sim} \sigma_{p, q}\left(\alpha_{2}\right)$, where $c=\alpha_{2} \beta_{1} /\left(\alpha_{1} \beta_{2}\right)$.

Proof. First, note that for any convex function $\varphi: \mathbb{R} \rightarrow \mathbb{R}$ we have

$$
\begin{aligned}
& \beta_{1} \varphi\left(-\alpha_{1} y\right)+\alpha_{1} \varphi\left(\beta_{1} y\right) \leq \beta_{2} \varphi\left(-\alpha_{2} \frac{\beta_{1}}{\beta_{2}} y\right)+\alpha_{2} \varphi\left(\beta_{2} \frac{\beta_{1}}{\beta_{2}} y\right), \\
& \beta_{2} \varphi\left(-\alpha_{2} y\right)+\alpha_{2} \varphi\left(\beta_{2} y\right) \leq \beta_{1} \varphi\left(-\alpha_{1} \frac{\alpha_{2}}{\alpha_{1}} y\right)+\alpha_{1} \varphi\left(\beta_{1} \frac{\alpha_{2}}{\alpha_{1}} y\right),
\end{aligned}
$$

for every $y \in \mathbb{R}$. For any fixed $u \in \mathbb{R}$ and $r \geq 1$ we consider the convex function $\varphi_{r}(x)=|1+x u|^{r}$. Putting $\sigma=\sigma_{p, q}\left(\alpha_{2}\right)$ and using (4.1), the definition of $\sigma_{p, q}$ and (4.2) we get

$$
\begin{aligned}
& \left(\beta_{1} \varphi_{p}\left(-\alpha_{1} \sigma\right)+\alpha_{1} \varphi_{p}\left(\beta_{1} \sigma\right)\right)^{1 / p} \leq\left(\beta_{2} \varphi_{p}\left(-\alpha_{2} \frac{\beta_{1}}{\beta_{2}} \sigma\right)+\alpha_{2} \varphi_{p}\left(\beta_{2} \frac{\beta_{1}}{\beta_{2}} \sigma\right)\right)^{1 / p} \\
& \leq\left(\beta_{2} \varphi_{q}\left(-\alpha_{2} \frac{\beta_{1}}{\beta_{2}}\right)+\alpha_{2} \varphi_{q}\left(\beta_{2} \frac{\beta_{1}}{\beta_{2}}\right)\right)^{1 / q} \leq\left(\beta_{1} \varphi_{q}\left(-c \alpha_{1}\right)+\alpha_{1} \varphi_{q}\left(c \beta_{1}\right)\right)^{1 / q}
\end{aligned}
$$

which proves $\sigma_{p, q}\left(\alpha_{2}\right) \leq c \sigma_{p, q}\left(\alpha_{1}\right)$, since $u \in \mathbb{R}$ can be chosen arbitrarily. Putting $\sigma=\sigma_{p, q}\left(\alpha_{1}\right)$ and using (4.2), the definition of $\sigma_{p, q}$ and (4.1) we have

$$
\begin{aligned}
& \left(\beta_{2} \varphi_{p}\left(-\alpha_{2} \sigma\right)+\alpha_{2} \varphi_{p}\left(\beta_{2} \sigma\right)\right)^{1 / p} \leq\left(\beta_{1} \varphi_{p}\left(-\alpha_{1} \frac{\alpha_{2}}{\alpha_{1}} \sigma\right)+\alpha_{1} \varphi_{p}\left(\beta_{1} \frac{\alpha_{2}}{\alpha_{1}} \sigma\right)\right)^{1 / p} \\
& \leq\left(\beta_{1} \varphi_{q}\left(-\alpha_{1} \frac{\alpha_{2}}{\alpha_{1}}\right)+\alpha_{1} \varphi_{q}\left(\beta_{1} \frac{\alpha_{2}}{\alpha_{1}}\right)\right)^{1 / q} \leq\left(\beta_{2} \varphi_{q}\left(-c \alpha_{2}\right)+\alpha_{2} \varphi_{q}\left(c \beta_{2}\right)\right)^{1 / q}
\end{aligned}
$$

which yields $\sigma_{p, q}\left(\alpha_{1}\right) \leq c \sigma_{p, q}\left(\alpha_{2}\right)$.

Lemma 4.2. For each $C>1$ there exists $D>1$ such that for all $1<q<$ $p \leq 2$ and $\alpha_{1}, \alpha_{2} \in(0,1 / 2]$, if $\alpha_{1} \stackrel{C}{\sim} \alpha_{2}$, then $\widetilde{\sigma}_{p, q}\left(\alpha_{1}\right) \stackrel{D}{\sim} \widetilde{\sigma}_{p, q}\left(\alpha_{2}\right)$.

Proof. For $\alpha_{1}, \alpha_{2} \in I_{j}(j=1, \ldots, 4)$ this is quite obvious-it suffices to observe that $\ln \left(1 / \alpha_{1}\right) \sim \ln \left(1 / \alpha_{2}\right)$ and in case $\alpha_{1}, \alpha_{2} \in I_{3}$ also that

$$
1+\ln \left(\frac{q-1}{p-1} \frac{1}{\alpha_{1}} \ln \frac{1}{\alpha_{1}}\right) \sim 1+\ln \left(\frac{q-1}{p-1} \frac{1}{\alpha_{2}} \ln \frac{1}{\alpha_{2}}\right) .
$$

To see the assertion for any pair $\alpha_{1}, \alpha_{2}$ satisfying the assumptions of the lemma, it suffices to show that $\widetilde{\sigma}_{p, q}(\alpha) \sim \lim _{\alpha^{\prime} \rightarrow \alpha^{+}} \widetilde{\sigma}_{p, q}\left(\alpha^{\prime}\right)$ for $\alpha=\sup I_{j}$ 
$(j=1,2,3)$. For $j=1$, we have

$$
\lim _{\alpha^{\prime} \rightarrow \alpha^{+}} \widetilde{\sigma}_{p, q}\left(\alpha^{\prime}\right)=\alpha^{1-1 / p} \sim \alpha^{1 / q-1 / p}=\widetilde{\sigma}_{p, q}(\alpha)
$$

since $\alpha^{1 / q-1}=e^{1 / q} \sim 1$. For $j=2$,

$$
\begin{aligned}
\lim _{\alpha^{\prime} \rightarrow \alpha^{+}} \tilde{\sigma}_{p, q}\left(\alpha^{\prime}\right) & =\frac{q-1}{p-1} \frac{\ln (1 / \alpha)}{1+\ln (1 /(p-1))+1 /(p-1)} \\
& \sim(q-1) \ln (1 / \alpha) \\
\widetilde{\sigma}_{p, q}(\alpha) & =(q-1) \ln (1 / \alpha)\left((q-1) \frac{1}{\alpha} \ln \frac{1}{\alpha}\right)^{1 / p-1} \\
& =(q-1) \ln (1 / \alpha) e^{-1 / p},
\end{aligned}
$$

and finally, for $j=3$ we have $\widetilde{\sigma}_{p, q}(\alpha)=\alpha=\lim _{\alpha^{\prime} \rightarrow \alpha^{+}} \widetilde{\sigma}_{p, q}\left(\alpha^{\prime}\right)$.

In the next two lemmas and in the proof of Theorem 2.1 we shall use the following notation. Let $1<q<p \leq 2, \alpha \in(0,1 / 2]$ with $\beta=1-\alpha$. For $x \in[-1 / \beta, 1 / \alpha]$, we define

$$
\begin{aligned}
L_{\alpha, p}(x) & =\beta(1-\alpha x)^{p}+\alpha(1+\beta x)^{p}-1 \\
R_{\alpha, q, p}(x) & =\left(\beta(1-\alpha x)^{q}+\alpha(1+\beta x)^{q}\right)^{p / q}-1 .
\end{aligned}
$$

If $\alpha \in\left(0, e^{-2}\right]$, we set

$$
\widetilde{L}_{\alpha, p}(x)= \begin{cases}(p-1) \alpha x^{2} & \text { for } x \in[0, e] \\ e(p-1) \alpha x \ln x & \text { for } x \in\left(e, e^{1 /(p-1)} \wedge 1 / \alpha\right], \\ \alpha x^{p} & \text { for } x \in\left(e^{1 /(p-1)} \wedge 1 / \alpha, 1 / \alpha\right],\end{cases}
$$

and, when additionally $1 / \alpha \notin\left(e^{1 /(q-1)}, e^{q /(q-1)}\right]$,

$$
\widetilde{R}_{\alpha, q, p}(x)=\left\{\begin{array}{ll}
(q-1) \alpha x^{2} & \text { for } x \in[0, e], \\
e(q-1) \alpha x \ln x & \text { for } x \in(e, 1 / \alpha],
\end{array} \quad \text { if } 1 / \alpha \leq e^{1 /(q-1)}\right.
$$

and

$\widetilde{R}_{\alpha, q, p}(x)= \begin{cases}(q-1) \alpha x^{2} & \text { for } x \in[0, e], \\ e(q-1) \alpha x \ln x & \text { for } x \in\left(e, e^{1 /(q-1)}\right], \\ \alpha x^{q} & \text { for } x \in\left(e^{1 /(q-1)},(1 / \alpha)^{1 / q}\right], \\ \alpha^{p / q} x^{p} & \text { for } x \in\left((1 / \alpha)^{1 / q}, 1 / \alpha\right],\end{cases}$

Note that $\widetilde{L}_{\alpha, p}$ and $\widetilde{R}_{\alpha, q, p}$ are continuous, differentiable (except the case $1 / \alpha>e^{q /(q-1)}$ when $\widetilde{R}_{\alpha, q, p}$ is not differentiable at $\left.(1 / \alpha)^{1 / q}\right)$ and increasing. 
Additionally, the following approximate formulas will be used: for every $\varepsilon>0$ there exist $c_{1}, c_{2}, c_{3}>0$ (each depends only on $\varepsilon$ ) such that

$$
\begin{aligned}
& e^{t}-1 \stackrel{c_{1}}{\sim} t \quad \text { for } 0 \leq t \leq \varepsilon, \\
& e^{t}-1 \stackrel{c_{2}}{\sim} e^{t} \quad \text { for } t \geq \varepsilon, \\
& \ln (1+t) \stackrel{c_{3}}{\sim} t \quad \text { for } 0 \leq t \leq \varepsilon .
\end{aligned}
$$

Lemma 4.3. For $\alpha \leq e^{-2}$ and $x \in[-1 / \beta, 1 / \alpha], \widetilde{L}_{\alpha, p}(|x|) \sim L_{\alpha, p}(x)$.

Proof. Obviously, for all $x_{1}, x_{2} \in[0,1 / \alpha]$ such that $x_{1} \stackrel{C}{\sim} x_{2}$ we have $\widetilde{L}_{\alpha, p}\left(x_{1}\right) \stackrel{D}{\sim} \widetilde{L}_{\alpha, p}\left(x_{2}\right)$, where $D>0$ depends only on $C$. Moreover, $L_{\alpha, p}$ is convex and $C^{\infty}$ on $(-1 / \beta, 1 / \alpha)$, hence $L_{\alpha, p}$ is decreasing on $[-1 / \beta, 0]$ and increasing on $[0,1 / \alpha]$, since $L_{\alpha, p}^{\prime}(0)=0$. Therefore it is sufficient to prove the assertion only for $x \in\{-1 / \beta\} \cup[-1 /(2 \beta), 1 /(2 \beta)] \cup\left[e^{2}, 1 / \alpha\right]$.

CASE $x \in[-1 /(2 \beta), 1 /(2 \beta)]$. It suffices to prove that

$$
L_{\alpha, p}^{\prime \prime}(x) \sim(p-1) \alpha .
$$

Indeed, by integrating we get $L_{\alpha, p}^{\prime}(x) \sim(p-1) \alpha x$, now, $L_{\alpha, p}^{\prime}(0)=0$, and by integrating once again we get $L_{\alpha, p}(x) \sim(p-1) \alpha x^{2}$, since $L_{\alpha, p}(0)=0$. Now,

$$
L_{\alpha, p}^{\prime \prime}(x)=p(p-1) \alpha \beta\left(\alpha(1-\alpha x)^{p-2}+\beta(1+\beta x)^{p-2}\right) .
$$

Clearly, we have $1-\alpha x \sim 1$ and $1+\beta x \sim 1$. Since $p-2 \in(-1,0]$, we also have $(1-\alpha x)^{p-2} \sim 1$ and $(1+\beta x)^{p-2} \sim 1$, which implies (4.7).

CASE $x=-1 / \beta$. From (4.4) and (4.6) we have

$$
\begin{aligned}
L_{\alpha, p}(-1 / \beta) & =\beta(1+\alpha / \beta)^{p}-1=(1+\alpha / \beta)^{p-1}-1=e^{(p-1) \ln (1+\alpha / \beta)}-1 \\
& \sim(p-1) \ln (1+\alpha / \beta) \sim(p-1) \alpha / \beta \sim \widetilde{L}_{\alpha, p}(1 / \beta),
\end{aligned}
$$

since $(p-1) \ln (1+\alpha / \beta) \leq(p-1) \alpha / \beta<1$ and $\alpha / \beta<1$.

CASE $x \in\left[e^{2}, 1 / \alpha\right]$. We shall approximate

$$
\begin{aligned}
L_{\alpha, p}(x) & =\beta(1-\alpha x)\left((1-\alpha x)^{p-1}-1\right)+\alpha(1+\beta x)\left((1+\beta x)^{p-1}-1\right) \\
& =M_{1}+M_{2} .
\end{aligned}
$$

The first term is equal to zero if $x=1 / \alpha$, and is negative otherwise. In the latter case we have the following estimate:

$$
\begin{aligned}
M_{1} & =\beta(1-\alpha x)\left(e^{(p-1) \ln (1-\alpha x)}-1\right) \geq \beta(1-\alpha x)(p-1) \ln (1-\alpha x) \\
& \geq \beta(p-1)(-\alpha x)>-(p-1) \alpha x
\end{aligned}
$$

where the second inequality follows from the inequality $\ln (1+y) \geq y /(1+y)$, which holds for $y>-1$. For the second term we have

$$
\begin{aligned}
M_{2} & =\alpha(1+\beta x)\left(e^{(p-1) \ln (1+\beta x)}-1\right) \geq \alpha(1+\beta x)(p-1) \ln (1+\beta x) \\
& \geq \alpha x(p-1) \ln x \geq 2(p-1) \alpha x,
\end{aligned}
$$


since $1+\beta x \geq x$ and $\ln x \geq 2$. Therefore $M_{2} \geq M_{1}+M_{2}=\left(M_{1}+M_{2} / 2\right)+$ $M_{2} / 2 \geq M_{2} / 2$, hence $L_{\alpha, p}(x) \sim M_{2}$ and further we deal with $M_{2}$. Since $1+\beta x \geq x \geq e^{2}$, we have $1+\beta x \sim x, \ln (1+\beta x) \sim \ln x$ and $(1+\beta x)^{p-1} \sim x^{p-1}$. Thus from (4.4) and (4.5) we obtain

$$
M_{2} \sim \alpha x\left(e^{(p-1) \ln (1+\beta x)}-1\right) \sim \alpha x \begin{cases}(p-1) \ln x & \text { if } x \leq e^{1 /(p-1)}, \\ x^{p-1} & \text { if } x>e^{1 /(p-1)} .\end{cases}
$$

Lemma 4.4. For all $x \in[-1 / \beta, 1 / \alpha], \widetilde{R}_{\alpha, q, p}(|x|) \sim R_{\alpha, q, p}(x)$, whenever $\widetilde{R}_{\alpha, q, p}$ is defined.

Proof. Since $p / q \in(1,2)$, we have $(1+t)^{p / q}-1 \sim(1+t)^{p / q} \sim t^{p / q}$ for $t \geq 1$, and $(1+t)^{p / q}-1 \sim t$ for $t \in[0,1]$. Therefore

$$
R_{\alpha, q, p}(x)=\left(1+L_{\alpha, q}(x)\right)^{p / q}-1 \sim L_{\alpha, q}(x) \vee L_{\alpha, q}(x)^{p / q},
$$

while an easy computation shows that $\widetilde{R}_{\alpha, q, p}(x) \sim \widetilde{L}_{\alpha, q}(x) \vee \widetilde{L}_{\alpha, q}(x)^{p / q}$. Then Lemma 4.3 completes the proof.

Proof of Theorem 2.1. To prove optimality of the constants $\widetilde{\sigma}_{p, q}(\alpha)$ we may proceed as follows. Let $p^{\prime}=p /(p-1), q^{\prime}=q /(q-1)$. Theorem 2.3 yields $\|S\|_{q^{\prime}} /\|S\|_{p^{\prime}} \sim \widetilde{\sigma}_{q^{\prime}, p^{\prime}}^{-1}(\alpha)$. Transforming $\left(q^{\prime}, p^{\prime}\right)$ to $(p, q)$ in the formula for $\widetilde{\sigma}_{q^{\prime}, p^{\prime}}(\alpha)$ from Theorem 2.2, together with Lemma 4.2, shows that $\widetilde{\sigma}_{q^{\prime}, p^{\prime}}(\alpha) \sim$ $\widetilde{\sigma}_{p, q}(\alpha)$. On the other hand, Proposition 1.1 implies that $\|S\|_{q^{\prime}} /\|S\|_{p^{\prime}} \leq$ $\sigma_{q^{\prime}, p^{\prime}}^{-1}(\alpha)=\sigma_{p, q}^{-1}(\alpha)$ (the last equality follows from Remark 1.1). Thus we get $\tilde{\sigma}_{p, q}(\alpha) \gtrsim \sigma_{p, q}(\alpha)$.

We proceed to show the converse inequality, i.e. $\widetilde{\sigma}_{p, q}(\alpha) \lesssim \sigma_{p, q}(\alpha)$. Lemmas 4.1 and 4.2 imply that we may neglect some values of $\alpha$, therefore we assume $\alpha \notin\left(e^{-2}, 1 / 2\right]$ and $1 / \alpha \notin\left(e^{1 /(q-1)}, e^{q /(q-1)}\right]$, since $e^{-2} \sim 1 / 2$ and $e^{1 /(q-1)} \sim e^{q /(q-1)}$. By Remark 1.2, it suffices to prove that

$$
L_{\alpha, p}(\sigma x) \leq R_{\alpha, q, p}(x) \quad \text { for all } x \in[-1 / \beta, 1 / \alpha],
$$

where $\sigma \sim \widetilde{\sigma}:=\widetilde{\sigma}_{p, q}(\alpha)$. This, in fact, can be reduced to showing that for some universal constant $C_{1}>0$,

$$
\widetilde{L}_{\alpha, p}(\widetilde{\sigma} x) \leq C_{1} \widetilde{R}_{\alpha, q, p}(x) \quad \text { for all } x \in[0,1 / \alpha] .
$$

Indeed, combining (4.9) with Lemmas 4.3 and 4.4 , we get

$$
L_{\alpha, p}(\widetilde{\sigma} x) \leq C_{2} R_{\alpha, q, p}(x) \quad \text { for all } x \in[-1 / \beta, 1 / \alpha],
$$

where $C_{2}$ is some universal constant. Clearly, we may assume $C_{2} \geq 1$. Since the left hand side of (4.10) is a convex function of $x$, taking the zero value at $x=0$, we have $L_{\alpha, p}\left(C_{2}^{-1} \widetilde{\sigma} x\right) \leq C_{2}^{-1} L_{\alpha, p}(\widetilde{\sigma} x)$, which combined with (4.10) gives (4.8) with $\sigma=C_{2}^{-1} \widetilde{\sigma}$.

We now claim that the continuous function $(0,1 / \alpha) \ni x \mapsto \frac{\widetilde{L}_{\alpha, p}(\widetilde{\sigma} x)}{\widetilde{R}_{\alpha, q, p}(x)}$ is non-decreasing. Since it is differentiable (except at the point $(1 / \alpha)^{1 / q}$ in case 
$\left.1 / \alpha>e^{q /(q-1)}\right)$, it suffices to show that its first derivative is non-negative, or, equivalently, that

$$
\widehat{L}_{\alpha, p}(\widetilde{\sigma} x) \geq \widehat{R}_{\alpha, q, p}(x) \quad \text { for all } x \in(0,1 / \alpha),
$$

(excluding $x=(1 / \alpha)^{1 / q}$ when $\left.1 / \alpha>e^{q /(q-1)}\right)$, where $\widehat{L}_{\alpha, p}(x)=x \frac{\widetilde{L}_{\alpha, p}^{\prime}(x)}{\widetilde{L}_{\alpha, p}(x)}$ and $\widehat{R}_{\alpha, q, p}(x)=x \frac{\widetilde{R}_{\alpha, q, p}^{\prime}(x)}{\widetilde{R}_{\alpha, q, p}(x)}$. Simple computations give

$$
\begin{aligned}
& \widehat{L}_{\alpha, p}(x)= \begin{cases}2 & \text { for } x \in(0, e], \\
1+1 / \ln x & \text { for } x \in\left(e, e^{1 /(p-1)} \wedge 1 / \alpha\right), \\
p & \text { for } x \in\left[e^{1 /(p-1)} \wedge 1 / \alpha, 1 / \alpha\right),\end{cases} \\
& \widehat{R}_{\alpha, q, p}(x)=\widehat{L}_{\alpha, p}(x) \quad \text { if } 1 / \alpha \leq e^{1 /(q-1)} \text {, } \\
& \widehat{R}_{\alpha, q, p}(x)=\left\{\begin{array}{ll}
2 & \text { for } x \in(0, e], \\
1+1 / \ln x & \text { for } x \in\left(e, e^{1 /(q-1)}\right], \\
q & \text { for } x \in\left(e^{1 /(q-1)},(1 / \alpha)^{1 / q}\right),
\end{array} \quad \text { if } 1 / \alpha>e^{q /(q-1)} .\right.
\end{aligned}
$$

$\widehat{L}_{\alpha, p}$ is non-increasing, thus $\widehat{L}_{\alpha, p}(\widetilde{\sigma} x) \geq \widehat{L}_{\alpha, p}(x)$, since $\widetilde{\sigma} \leq 1$ (this can be checked in many ways, e.g. see the proof of Lemma 2.2, where monotonicity of $\alpha \mapsto \widetilde{\sigma}_{p, q}(\alpha)$ is investigated). Moreover, $\widehat{L}_{\alpha, p}(x)=\widehat{R}_{\alpha, q, p}(x)$ for $x \in$ $\left(0, e^{1 /(p-1)} \wedge 1 / \alpha\right)$ and $\widehat{L}_{\alpha, p}(x)=p \geq \widehat{R}_{\alpha, q, p}(x)$ for $x \in\left[e^{1 /(p-1)} \wedge 1 / \alpha, 1 / \alpha\right)$ (excluding $x=(1 / \alpha)^{1 / q}$ when $\left.1 / \alpha>e^{q /(q-1)}\right)$, which proves (4.11).

To complete the proof, it remains to check (4.9) for $x=1 / \alpha$. We consider four cases (as in the formula for $\widetilde{\sigma}_{p, q}(\alpha)$ ).

CASE $\alpha \in I_{1}$. Clearly $\widetilde{\sigma} / \alpha=(1 / \alpha)^{1-1 / q+1 / p}>(1 / \alpha)^{1 / p}$. Since we assumed that $1 / \alpha \notin\left(e^{1 /(q-1)}, e^{q /(q-1)}\right]$, we have $1 / \alpha>e^{q /(q-1)}>e^{p /(p-1)}$, and therefore $\widetilde{\sigma} / \alpha>e^{1 /(p-1)}$, hence $\widetilde{L}_{\alpha, p}(\widetilde{\sigma} / \alpha)=\alpha(\widetilde{\sigma} / \alpha)^{p}=(1 / \alpha)^{p-p / q}=$ $\widetilde{R}_{\alpha, q, p}(1 / \alpha)$.

CASE $\alpha \in I_{2}$. Clearly, $\widetilde{R}_{\alpha, q, p}(1 / \alpha)=e(q-1) \ln (1 / \alpha)$. If $\widetilde{\sigma} / \alpha>e^{1 /(p-1)}$, then $\widetilde{L}_{\alpha, p}(\widetilde{\sigma} / \alpha)=\alpha(\widetilde{\sigma} / \alpha)^{p}=(q-1) \ln (1 / \alpha)$, and if $\widetilde{\sigma} / \alpha \leq e^{1 /(p-1)}$, then by the monotonicity of $\widetilde{L}_{\alpha, p}(x)$ and $x \mapsto x \ln x$ we have

$$
\widetilde{L}_{\alpha, p}(\widetilde{\sigma} / \alpha) \leq e(p-1) \alpha e^{1 /(p-1)} \ln \left(e^{1 /(p-1)}\right)=\alpha e^{p /(p-1)} \leq(q-1) \ln (1 / \alpha),
$$

where the last inequality is due to the fact that $\alpha \in I_{2}$.

CASE $\alpha \in I_{3}$. $\widetilde{R}_{\alpha, q, p}(1 / \alpha)$ is the same as in the previous case. We now show that $\tilde{\sigma} / \alpha \leq e^{1 /(p-1)}$. Setting

$$
u_{0}:=\ln \left(\frac{q-1}{p-1} \frac{1}{\alpha} \ln \frac{1}{\alpha}\right),
$$


we get $\widetilde{\sigma} / \alpha=e^{u_{0}} /\left(1+u_{0}\right)$. Since $\alpha \in I_{3}$, we have

$$
0 \leq u_{0} \leq \frac{1}{p-1}+\ln \frac{1}{p-1} \text {. }
$$

The function $h(u)=e^{u} /(1+u)$ is increasing for $u \geq 0\left(h^{\prime}(u)=u e^{u} /(1+u)^{2}\right)$, hence

$$
\widetilde{\sigma} / \alpha \leq h\left(\frac{1}{p-1}+\ln \frac{1}{p-1}\right)<e^{1 /(p-1)} .
$$

We next estimate the fraction $\widetilde{L}_{\alpha, p}(\widetilde{\sigma} / \alpha) / \widetilde{R}_{\alpha, q, p}(1 / \alpha)$. If $\widetilde{\sigma} / \alpha>e$, then $\widetilde{L}_{\alpha, p}(\tilde{\sigma} / \alpha)=e(p-1) \widetilde{\sigma} \ln (\widetilde{\sigma} / \alpha)$, otherwise, by the monotonicity of $\widetilde{L}_{\alpha, p}(x)$, $\widetilde{L}_{\alpha, p}(\tilde{\sigma} / \alpha) \leq(p-1) \alpha e^{2}$. In the first case, the fraction above is equal to

$$
\frac{e(p-1) \widetilde{\sigma} \ln (\widetilde{\sigma} / \alpha)}{e(q-1) \ln (1 / \alpha)}=\frac{u_{0}+\ln \frac{1}{1+u_{0}}}{1+u_{0}}<1 .
$$

In the second case the same fraction is equal to

$$
\frac{(p-1) \alpha e^{2}}{e(q-1) \ln (1 / \alpha)} \leq e, \quad \text { since } \quad \frac{p-1}{q-1} \leq \frac{1}{\alpha} \ln \frac{1}{\alpha}
$$

(because $\alpha \in I_{3}$ ).

CASE $\alpha \in I_{4} . \widetilde{R}_{\alpha, q, p}(1 / \alpha)$ is as in the previous case. Clearly, $\widetilde{\sigma} / \alpha \in(0,1)$, thus

$$
\frac{\widetilde{L}_{\alpha, p}(\widetilde{\sigma} / \alpha)}{\widetilde{R}_{\alpha, q, p}(1 / \alpha)}=\frac{(p-1) \widetilde{\sigma}^{2} / \alpha}{e(q-1) \ln (1 / \alpha)}=e^{-1} .
$$

This gives (4.9) with the constant $C_{1}=e$, which completes the proof.

Proof of Theorem 2.2. At the beginning of the proof of Theorem 2.1 we stated that $\widetilde{\sigma}_{p, q}(\alpha) \sim \widetilde{\sigma}_{q^{\prime}, p^{\prime}}(\alpha)$ for $p^{\prime}=p /(p-1), q^{\prime}=q /(q-1)$, whereas Theorem 2.1 and Remark 1.1 imply that $\widetilde{\sigma}_{q^{\prime}, p^{\prime}}(\alpha) \sim \sigma_{q^{\prime}, p^{\prime}}(\alpha)=\sigma_{p, q}(\alpha)$.

Proof of Lemma 2.2. By Remark 1.1, we can assume $1<q<p \leq 2$. Theorem 2.1 implies that it suffices to prove $\widetilde{\sigma}_{p, q}\left(\alpha_{1}\right) \lesssim \widetilde{\sigma}_{p, q}\left(\alpha_{2}\right)$, and Lemma 4.2 shows that it suffices to do it only for $\alpha_{1}, \alpha_{2} \in I_{j}, j=1, \ldots, 4$.

CASE $\alpha_{1}, \alpha_{2} \in I_{1}$. The function $\alpha \mapsto \alpha^{1 / q-1 / p}$ is increasing, since $1 / q-$ $1 / p>0$.

CASE $\alpha_{1}, \alpha_{2} \in I_{2}$. We check that the function $h(\alpha)=(\ln (1 / \alpha))^{1 / p} \alpha^{1-1 / p}$ is increasing for $\alpha \leq e^{-1 /(p-1)}$, so in particular for $\alpha \in I_{2}$. Indeed,

$$
\begin{aligned}
h^{\prime}(\alpha) & =-\frac{1}{p}(\alpha \ln (1 / \alpha))^{-1} h(\alpha)+\left(1-\frac{1}{p}\right) \alpha^{-1} h(\alpha) \\
& =\alpha^{-1} h(\alpha)\left(1-\frac{1}{p}\left(1+(\ln (1 / \alpha))^{-1}\right)\right)
\end{aligned}
$$

which is non-negative, since $1+(\ln (1 / \alpha))^{-1} \leq p$. 
CASE $\alpha_{1}, \alpha_{2} \in I_{3}$. We check that the function

$$
h(\alpha)=\frac{h_{1}(\alpha)}{1+\ln h_{2}(\alpha)}=\frac{\ln (1 / \alpha)}{1+\ln \left(\frac{q-1}{p-1} \frac{1}{\alpha} \ln \frac{1}{\alpha}\right)}
$$

is increasing if $\ln (1 / \alpha) \leq(p-1) /(q-1)$ and decreasing otherwise. Indeed, the sign of its first derivative is the same as the sign of

$$
\begin{aligned}
& h_{1}^{\prime}(\alpha)\left(1+\ln h_{2}(\alpha)\right)-h_{1}(\alpha) \frac{h_{2}^{\prime}(\alpha)}{h_{2}(\alpha)} \\
& =-\alpha^{-1}\left(1+\ln h_{2}(\alpha)\right)+h_{1}(\alpha) \frac{1+\ln (1 / \alpha)}{\alpha \ln (1 / \alpha)} \\
& =-\alpha^{-1}\left(\ln h_{2}(\alpha)-\ln (1 / \alpha)\right)=-\alpha^{-1} \ln \left(\frac{q-1}{p-1} \ln \frac{1}{\alpha}\right) .
\end{aligned}
$$

Thus if $\{\alpha: \ln (1 / \alpha)>(p-1) /(q-1)\} \cap I_{3}=\emptyset$, then this case is done. Assume the opposite. Then we have the non-empty interval $(\underline{\alpha}, \bar{\alpha}]$ (where $\underline{\alpha}=\inf I_{3}$ and $\left.\ln (1 / \bar{\alpha})=(p-1) /(q-1)\right)$ on which $h(\alpha)$ is decreasing. So $\underline{\alpha}<\bar{\alpha}$, which implies that

so

$$
\frac{p-1}{q-1} e^{(p-1) /(q-1)}<\frac{1}{q-1} e^{1 /(p-1)}
$$

hence

$$
\frac{1}{q-1}<\frac{1}{(p-1)^{2}}+\frac{1}{p-1} \ln \frac{1}{p-1}<\frac{2}{(p-1)^{2}}
$$

$$
\ln \frac{1}{q-1}<\ln 2+2 \ln \frac{1}{p-1}<\frac{3}{p-1} .
$$

It suffices to show that for some universal constant $C \geq 1$ we have $\lim _{\alpha \rightarrow \underline{\alpha}^{+}} \widetilde{\sigma}_{p, q}(\alpha) \leq C \widetilde{\sigma}_{p, q}(\bar{\alpha})$. Clearly,

$$
\widetilde{\sigma}_{p, q}(\bar{\alpha})=\frac{1}{1+\frac{p-1}{q-1}} \sim \frac{q-1}{p-1}
$$

and from (4.3) we have

since

$$
\lim _{\alpha \rightarrow \underline{\alpha}^{+}} \widetilde{\sigma}_{p, q}(\alpha) \sim(q-1) \ln \frac{1}{\underline{\alpha}} \sim(q-1)\left(\frac{1}{p-1}+\ln \frac{1}{q-1}\right),
$$

Hence

$$
\ln \frac{1}{\underline{\alpha}} \sim \ln \frac{e^{1 /(p-1)}}{q-1} .
$$

$$
\lim _{\alpha \rightarrow \underline{\alpha}^{+}} \widetilde{\sigma}_{p, q}(\alpha) \sim \frac{q-1}{p-1}, \quad \text { since } \quad \ln \frac{1}{q-1} \lesssim \frac{1}{p-1} .
$$

CASE $\alpha_{1}, \alpha_{2} \in I_{4}$. We check that the function $\alpha \mapsto \alpha \ln (1 / \alpha)$ is increasing for $\alpha \leq e^{-1}$. Indeed, its first derivative is $\ln (1 / \alpha)-1$. By Lemma 4.2 we have $\widetilde{\sigma}_{p, q}(\alpha) \sim \widetilde{\sigma}_{p, q}\left(e^{-1}\right)$ for $\alpha \in\left(e^{-1}, 1 / 2\right]$, so this case is done. 
Proof of Theorem 2.3. Let $\left(X_{k}^{\prime}\right)_{k=1}^{\infty}$ be an independent copy of the sequence $\left(X_{k}\right)_{k=1}^{\infty}$. We set $Y_{k}=X_{k}-X_{k}^{\prime}, S^{\prime}=\sum_{k=1}^{n(\alpha, p)} X_{k}^{\prime}$ and $S^{\prime \prime}=S-S^{\prime}$ and $X=X_{1}, Y=Y_{1}$. Each random variable $Y_{k}$ is symmetric and its distribution is equal to $\alpha \beta \delta_{-1}+(1-2 \alpha \beta) \delta_{0}+\alpha \beta \delta_{1}$. Moreover, for any $r \geq 1$ we have $\|X\|_{r} \leq\|Y\|_{r} \leq 2\|X\|_{r}$ (the first inequality comes from the Jensen inequality and the fact that $E X=0$, whereas the second is just the triangle inequality for the $L_{r}$-norm). Clearly, the same applies to $S$ and $S^{\prime \prime}$. Therefore we proceed to show that $\left\|S^{\prime \prime}\right\|_{q} /\left\|S^{\prime \prime}\right\|_{p} \sim \widetilde{\sigma}_{p, q}(\alpha)$.

First, note that if $p \leq \ln (1 / \alpha)$ (equivalently $n(\alpha, p)=1$ ), then $\alpha \in I_{1}^{\prime}$ and we have $\left\|S^{\prime \prime}\right\|_{q}=\|Y\|_{q}=(2 \alpha \beta)^{1 / q} \sim \alpha^{1 / q}$ and $\left\|S^{\prime \prime}\right\|_{p} \sim \alpha^{1 / p}$.

Further we assume that $p>\ln (1 / \alpha)$ (equivalently, $n:=n(\alpha, p) \geq 2$ ). It is a simple matter to approximate $\left\|S^{\prime \prime}\right\|_{p}$. Indeed, we have $n / 2<p / \ln (1 / \alpha)$, thus $e^{-4 p}<\alpha^{2 n}<(\alpha \beta)^{n}$, so $P\left(S^{\prime \prime}=n\right)>e^{-4 p}$. Hence

$$
n=\left\|S^{\prime \prime}\right\|_{\infty} \geq\left\|S^{\prime \prime}\right\|_{p} \geq n P\left(S^{\prime \prime}=n\right)^{1 / p}>n e^{-4},
$$

so $\left\|S^{\prime \prime}\right\|_{p} \sim n \sim p / \ln (1 / \alpha)$. We now want to approximate $\left\|S^{\prime \prime}\right\|_{q}$. Originally the author just used an explicit approximate formula due to Rafał Latała [unpublished]. We may also apply [8, Corollary 2] which states that for $q \geq 2$ and i.i.d. symmetric random variables $Z, Z_{1}, \ldots, Z_{n}$,

$$
\left\|Z_{1}+\cdots+Z_{n}\right\|_{q} \sim \sup \left\{\frac{q}{s}\left(\frac{n}{q}\right)^{1 / s}\|Z\|_{s}: 2 \vee \frac{q}{n} \leq s \leq q\right\} .
$$

In our situation, we have

$$
\frac{\left\|S^{\prime \prime}\right\|_{q}}{\left\|S^{\prime \prime}\right\|_{p}} \sim \bar{f}:=\sup \left\{f(s)=\frac{1}{s}\left(\frac{q}{p} \ln \frac{1}{\alpha}\right)^{1-1 / s} \alpha^{1 / s}: 2 \vee \frac{q}{\left\lceil\frac{p}{\ln (1 / \alpha)}\right\rceil} \leq s \leq q\right\},
$$

since $n^{1 / s} \sim(p / \ln (1 / \alpha))^{1 / s}$. Note that $f$ is differentiable for $s \in(0, \infty)$ and

$$
f^{\prime}(s)=\left(\frac{q}{p} \ln \frac{1}{\alpha}\right)\left(\frac{1}{s}\left(\frac{q}{p} \frac{1}{\alpha} \ln \frac{1}{\alpha}\right)^{-1 / s}\right)^{\prime}=\frac{1}{s^{2}} f(s)\left(\ln \left(\frac{q}{p} \frac{1}{\alpha} \ln \frac{1}{\alpha}\right)-s\right),
$$

hence $f(s)$ is increasing for $0<s<s_{0}$ and decreasing for $s_{0}<s<\infty$, where $s_{0}=\ln \left(\frac{q}{p} \frac{1}{\alpha} \ln \frac{1}{\alpha}\right)$. Let us consider three cases:

$\operatorname{CASE}(p / q) e^{q} \leq(1 / \alpha) \ln (1 / \alpha)<p e^{p}$. Clearly, $s_{0} \geq q$, hence

$$
\bar{f}=f(q)=q^{-1 / q}\left(\frac{\ln (1 / \alpha)}{p}\right)^{1-1 / q} \alpha^{1 / q} \sim\left(\frac{\ln (1 / \alpha)}{p}\right)^{1-1 / q} \alpha^{1 / q} .
$$

If $p e^{q} \leq(1 / \alpha) \ln (1 / \alpha)<p e^{p}$ (i.e. $\left.\alpha \in I_{2}^{\prime}\right)$, then we have $\bar{f} \sim \widetilde{\sigma}_{p, q}(\alpha)$. If $(p / q) e^{q} \leq(1 / \alpha) \ln (1 / \alpha)<p e^{q}$ (hence $\left.\alpha \in I_{3}^{\prime}\right)$, then we see that

$$
\frac{\widetilde{\sigma}_{p, q}(\alpha)}{\left(\frac{\ln (1 / \alpha)}{p}\right)^{1-1 / q} \alpha^{1 / q}}=\frac{q}{1+\ln \left(\frac{q}{p} \frac{1}{\alpha} \ln \frac{1}{\alpha}\right)}\left(\frac{1}{p} \frac{1}{\alpha} \ln \frac{1}{\alpha}\right)^{1 / q} \sim 1
$$


since the first term is $\sim 1$ because $q \leq \ln \left(\frac{q}{p} \frac{1}{\alpha} \ln \frac{1}{\alpha}\right)<q+\ln q$, as also is the second, because $q^{-1} e^{q} \leq \frac{1}{p} \frac{1}{\alpha} \ln \frac{1}{\alpha}<e^{q}$, so this case is done.

$\operatorname{CASE}(p / q) e^{2} \leq(1 / \alpha) \ln (1 / \alpha)<(p / q) e^{q}$. First, we show that

$$
2 \vee \frac{q}{\lceil p / \ln (1 / \alpha)\rceil} \leq s_{0}<q
$$

It is clear that $2 \leq s_{0}<q$. If $s_{0}<q /\lceil p / \ln (1 / \alpha)\rceil$, we would have $s_{0}<$ $(q / p) \ln (1 / \alpha)$, hence by monotonicity of $\ln$ we would obtain

$$
\ln \frac{1}{\alpha}<\ln \frac{2}{\alpha}<\ln \left(\frac{q}{p} \frac{1}{\alpha} \ln \frac{1}{\alpha}\right)=s_{0}<\frac{q}{p} \ln \frac{1}{\alpha},
$$

which is a contradiction, since $q<p$. Therefore $\bar{f}=f\left(s_{0}\right)$. We now show that $f\left(s_{0}\right) \sim \tilde{\sigma}_{p, q}(\alpha)$. Since $s_{0} \geq 2$, we have $s_{0} \sim 1+s_{0}$, so

$$
f\left(s_{0}\right) \sim \frac{q}{p} \frac{\ln (1 / \alpha)}{1+s_{0}}\left(\frac{q}{p} \frac{1}{\alpha} \ln \frac{1}{\alpha}\right)^{-1 / s_{0}}=\widetilde{\sigma}_{p, q}(\alpha)\left(e^{s_{0}}\right)^{-1 / s_{0}} \sim \widetilde{\sigma}_{p, q}(\alpha) .
$$

$\operatorname{CASE}(1 / \alpha) \ln (1 / \alpha)<(p / q) e^{2}$. In this case $s_{0}<2$ and

$$
\frac{q}{\lceil p / \ln (1 / \alpha)\rceil} \leq \frac{q}{p} \ln \frac{1}{\alpha}<\alpha e^{2} .
$$

If $\alpha e^{2} \leq 2$, then $q /\lceil p / \ln (1 / \alpha)\rceil<2$. Hence in the case $\alpha \leq 2 e^{-2}$ or $\alpha \in$ $\left(2 e^{-2}, 1 / 2\right]$ and $q /\lceil p / \ln (1 / \alpha)\rceil \leq 2$ we have $\bar{f}=f(2)=\frac{1}{2} \sqrt{(q / p) \alpha \ln (1 / \alpha)}$, which is $\sim \widetilde{\sigma}_{p, q}(\alpha)$ if $\alpha \in I_{4}^{\prime}$. If $\alpha \in I_{3}^{\prime}$, i.e. $p / q \leq(1 / \alpha) \ln (1 / \alpha)<(p / q) e^{2}$, then by Lemma 4.2 (more precisely: by an analogous fact which deals with the dual case $2 \leq q<p<\infty)$ we get $\bar{f} \sim \alpha \sim \widetilde{\sigma}_{p, q}(\alpha)$. There remains the case when $\alpha \in\left(2 e^{-2}, 1 / 2\right]$ and $s_{1}:=q /\lceil p / \ln (1 / \alpha)\rceil>2$. Then $\bar{f}=f\left(s_{1}\right)$ and $2<s_{1} \leq(q / p) \ln (1 / \alpha)<\alpha e^{2} \leq \frac{1}{2} e^{2}$. Thus $\bar{f} \sim 1$ and $q / p \sim 1$, since $\ln (1 / \alpha) \sim 1$. The latter implies that $\widetilde{\sigma}_{p, q}(1 / 2) \sim 1$ and from Lemma 4.2 we know that $\widetilde{\sigma}_{p, q}(\alpha) \sim \widetilde{\sigma}_{p, q}(1 / 2)$.

Acknowledgments. The author wishes to express his thanks to Prof. Krzysztof Oleszkiewicz for suggesting the problem and for many stimulating conversations. The author is also indebted to Prof. Rafał Latała for sharing very useful unpublished results.

\section{References}

[1] W. Beckner, Inequalities in Fourier analysis, Ann. of Math. 102 (1975), 159-182.

[2] A. Bonami, Étude des coefficients de Fourier des fonctions de $L^{p}(G)$, Ann. Inst. Fourier (Grenoble) 20 (1970), no. 2, 335-402.

[3] P. Diaconis and L. Saloff-Coste, Logarithmic Sobolev inequalities for finite Markov chains, Ann. Appl. Probab. 6 (1996), 695-750.

[4] S. Janson, Hypercontractivity and large deviations for functions of discrete random variables, in preparation. 
[5] W. Krakowiak and J. Szulga, Hypercontraction principle and random multilinear forms, Probab. Theory Related Fields 77 (1988), 325-342.

[6] S. Kwapień and J. Szulga, Hypercontraction methods in moment inequalities for series of independent random variables in normed spaces, Ann. Probab. 19 (1991), 369-379.

[7] S. Kwapień and W. A. Woyczyński, Random Series and Stochastic Integrals: Single and Multiple, Birkhäuser, New York, 1992.

[8] R. Latała, Estimation of moments of sums of independent real random variables, Ann. Probab. 25 (1997), 1502-1513.

[9] R. Latała and K. Oleszkiewicz, Between Sobolev and Poincaré, in: Geometric Aspects of Functional Analysis, Lecture Notes in Math. 1745, Springer, Berlin, 2000, $147-168$.

[10] K. Oleszkiewicz, On a nonsymmetric version of the Khinchine-Kahane inequality, in: Stochastic Inequalities and Applications, Progr. Probab. 56, Birkhäuser, Basel, 2003, 157-168.

[11] O. Rothaus, Logarithmic Sobolev inequalities and the spectrum of Sturm-Liouville operators, J. Funct. Anal. 39 (1980), 42-56.

Institute of Mathematics

Warsaw University

Banacha 2

02-097 Warszawa, Poland

E-mail: pwolff@mimuw.edu.pl

Received January 7, 2005

Revised version February 15, 2007 